\title{
Temperature dependent growth rate of phytoplankton and salinity induced grazing rate of zooplankton as determinants of realistic multi-delayed food chain model
}

\author{
Ujjwal Roy $^{1}$ N. C. Majee ${ }^{1} \cdot$ Santanu Ray ${ }^{2}$
}

Received: 5 July 2016/Accepted: 13 August 2016/Published online: 31 August 2016

(C) Springer International Publishing Switzerland 2016

\begin{abstract}
Impact of temperature dependent growth rate of phytoplankton, salinity induced grazing rate of zooplankton and gestation delay of phytoplankton and zooplankton differently are considered in the present study. Reproduction of predator after consuming prey is not instantaneous, but mediated by some time lag required for gestation. Here first gestation delay is considered in the numerical response term of phytoplankton and second one is at the numerical response term of zooplankton respectively. There is a very interesting interplay between these two delays. In accordance with previous studies, it is observed that delay destabilizes the system, in general and stability loss occurs via Hopf-bifurcation. In particular, we show that there exists critical values of the delay parameters below which the coexistence equilibrium is stable and above which it is unstable. Hopf bifurcation occurs when the delay parameters cross their critical values. All the stability results of the system significantly depend upon the temperature and salinity of the environment. Numerical examples are also support the model assumption and analytical results.
\end{abstract}

Keywords Temperature dependent growth rate $\cdot$ Salinity induced grazing rate $\cdot$ Gestation delay $\cdot$ Multiple delays . Hopf-bifurcation

Ujjwal Roy

ujjwal2ju@gmail.com

1 Department of Mathematics, Jadavpur University, Kolkata 700 032, India

2 Ecological Modelling Laboratory, Department of Zoology, Visva Bharati University, Santiniketan 731 235, India

\section{Introduction}

Understanding the dynamical relationship between resource and consumer through mathematical modeling in theoretical ecology has gained a lot of importance during the last few decades due to its universal existence and importance (Berryman 1992). Different food chain models are studied by many authors (Hassell 1971; Jana et al. 2014). Dynamical complexity arises in food chain model through the consideration of functional response of consumer. Consumer's (predator's) functional response which is defined as the amount of resource (prey) catch per consumer (predator) per unit of time is assumed to be one of the most important components of resource-consumer/prey-predator interaction because predation intensity may change the shape of the community structure and ecosystem properties (Sih et al. 1985). A brief review on prey-predator models with discrete delay for both Kolmogorov and Non-Kolmogorov type model system is presented by Ruan (2009) where he shows different mathematical criterions for different biological assumptions due to delay. Models with delay are much more realistic. In reality time delays occur in almost every biological situation (Jana et al. 2015; MacDonald 1989; Jana et al. 2014) and assumed to be one of the significant reasons of regular fluctuations in population density (Jana et al. 2015; MacDonald 1989; Jana et al. 2014; May 1981). Reproduction of predator after consuming prey is not instantaneous, but mediated by some time lag required for gestation. Therefore, to make a prey-predator model biologically more realistic, one has to consider this gestation delay in the model system. In food chain models, different consumers show different gestation time, so it is very important and significant observation to study impact of multiple time lags as gestation delays of different consumers in one food chain model. Jana (2014) studies the 
stabilizing effect of prey refuge and predator's interference on the dynamics of prey with delayed growth and generalist predator with delayed gestation. Jana and Samanta (2014) show the complex dynamical results of a multi-delayed ratio-dependent prey-predator system with prey harvesting under stochastic environment. Where as Jana et al. (2015) show the effect of multiple delays on the dynamics of generalist predator in a stochastic environment. Effect of age-selective harvesting of both prey and predator is studied by Jana et al. (2016).

Temperature plays a significant role to maintain the phytoplankton population at estuary because the temperature directly affects the rates of many reactions including photosynthesis and respiration (cf. Lomas et al. 2002) and indirectly affects phytoplankton through the activity of grazer (cf. Smayda 2004). The growth of phytoplankton species has an optimal temperature range (cf. Zhou 2006). Bintz et al. (2003) shows the phytoplankton blooms are completely absent from cool un-enriched systems and warmer systems promote phytoplankton blooms. Salinity is one of the most important factors for governing zooplankton population dynamics of any estuary (cf. Ghosh 2001; Ketchum 1951) which defines estuaries in such a way as to include any marine waters in which seawater was measurably diluted with fresh water from land drainage. Dube et al. (2010) conclude that salinity is uniform through the water column but varies between the riverine region (1 psu) and seaside (30 psu) in shallow lagoons with no distinct stratification. Growth models by using light and temperature effects on phytoplankton are developed by Baird et al. (2001), Flynn (2003), and Bissinger et al. (2008). Baird et al. (2001) use the interacting effects of temperature, light capture and nutrient uptake on phytoplankton growth model. Bissinger et al. (2008) uses quantile regression method and improve Eppley curve to detect the maximum growth rates of marine phytoplankton as a function of temperature. Different factors such as temperature (cf. Di Toro et al. 1975; Nicklisch et al. 2008; Sipkay 2010), body size [(cf. Ray et al. 2001), nutrient pulse (cf. Cottingham et al. 2004; Dube and Jayaraman 2008; Dube et al. 2010), top-down effect (cf. Morozov et al. 2005; Irigoien et al. 2005; Calbet and Saiz 2005)] are included in the NPZ models to know the effects of these factors on plankton dynamics.

In the present work, we have investigated the complex dynamical behavior of a nutrient-phytoplankton-zooplankton population with two delays both describes the gestation period of phytoplankton and zooplankton respectively. The paper is structured as follows: in "Mathematical model formulation", study area, the model system, its stability and Hopf-bifurcation has been discussed, numerical simulation results have been presented in
"Numerical simulation" and at last, in "Discussion", conclusions and discussions have been made.

\section{Mathematical model formulation}

\section{Study area}

The total length of Hooghly estuary is about $295 \mathrm{~km}$ and it lies between the latitude $21^{\circ} 31^{\prime} \mathrm{N}$ to $23^{\circ} 30^{\prime} \mathrm{N}$ and $87^{\circ} 45^{\prime} \mathrm{N}$ to $88^{\circ} 45^{\prime} \mathrm{N}$. The estuary bifurcates near Sagar island into main Hooghly in the north and northwestern side and river Mooriganga in the eastern side, which is connected to another river Thakuran and Matla, forming the Sundarban estuarine complex. The present study is restricted to mangrove dominated region (lower part) of the estuarine complex. The creeks traversing these regions belong to shallow, mesotidal regime (Fig. 1).

The seasons of this region are categorized as pre-monsoon (March-June), monsoon (July-October) and postmonsoon (November-February). The pre-monsoon is characterized by high air temperature ranging from 28 to $40^{\circ} \mathrm{C}$ and occasional rains. The monsoon encounters high rainfall (average $185 \mathrm{cms}$.), when the south west wind enters the Indian subcontinent. The post-monsoon is

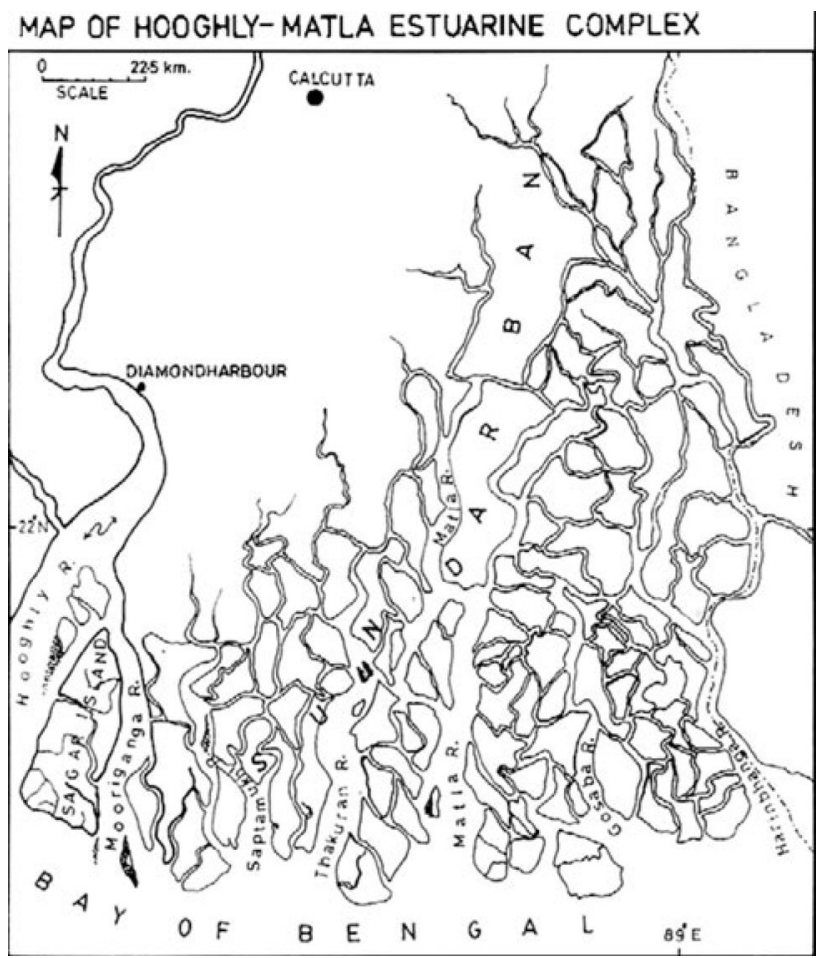

Fig. 1 Map showing the study area in Hooghly-Matla estuarine complex 
characterized by cold weather (average $22^{\circ} \mathrm{C}$ ) and negligible rainfall.

\section{Model and analysis}

Now we consider a $N P Z$ model in the given form where $N, P$ and $Z$ represent nutrient, phytoplankton and zooplankton densities respectively.

$$
\begin{aligned}
& \frac{d N}{d t}=I n-N L_{r}-\delta \frac{P N}{N+K_{p}}, \\
& \frac{d P}{d t}=\delta \frac{P N}{N+K_{p}}-P r_{1}-g_{s} \frac{P Z}{P+K_{Z}}, \\
& \frac{d Z}{d t}=g_{s} \frac{P Z}{P+K_{Z}}-Z r_{2} .
\end{aligned}
$$

In this present $N P Z$ model temperature dependant phytoplankton growth rate and salinity induced grazing rate of zooplankton are incorporated this equation which are taken from the model of Mandal et al. (2012). In the present model, Dissolved Inorganic Nitrogen is considered as nutrient $(N)$ for phytoplankton. In $=$ input of nutrient through mineralization and leaching, $L_{r}, K_{p}, N, P, \delta$ are respectively loss rate of nutrient, half saturation constant for nutrient uptake by phytoplankton, nutrient, phytoplankton, temperature dependent phytoplankton growth rate.

$\delta=\frac{P_{\max } e^{\gamma T} L_{e f f}}{\alpha}$,

where $P_{\max }, T, L_{\text {eff }}$ are respectively maximum growth rate of Phytoplankton, water temperature, light effect on photosynthesis. Phytoplankton growth rate is $T$ dependent and the dependence of $T$ is exponential. $\gamma=0.09$ and $\alpha=6.05$ is define by Ray and Straskraba (2001).

$$
L_{\text {eff }}=\frac{I}{I_{\text {opt }}} e^{1-\frac{I}{I_{\text {opt }}}}
$$

where $I=$ irradiance effect, $I_{o p t}$ is the optimum surface solar radiation for photosynthesis.

$I=I_{r} e^{\left(-E_{c}-E_{x x}\right) d}$,

where $I_{r}, E_{c}, d, E_{x t}$, are respectively surface solar irradiance, extinction coefficient of water, depth of water and self shading of phytoplankton due to its own body size. Self shading is calculated by using the equation of Radtke and Straskraba (1980). The equation is based on the physical laws of particle sizes and as follows:

$E_{x t}=0.12 V^{(-0.33)}$,

where $E_{x t}, V$ are respectively self shading of phytoplankton due to its own body size and average body volume of phytoplankton. $r_{1}=\left(r_{p h}+r_{s}+M_{r p}\right)$,

where $r_{p h}, r_{s}, M_{r p}$ are respectively respiratory rate of phytoplankton, rate of settling, mortality rate of phytoplankton.

$g_{s}=g_{z} \frac{s_{e}}{s_{e}-s_{r}}$,

where, $g_{s}, S_{r}, S_{e}, g_{z}, S_{r}, K_{z}$, are respectively salinity induced grazing of zooplankton, salinity of upstream, salinity of downstream, grazing rate of zooplankton, Half saturation constant of zooplankton on phytoplankton grazing by zooplankton.

$r_{2}=\left(r_{z o}+r_{f p}+M_{r z}\right)$,

where $r_{f p}, r_{z o}, M_{r z}$, are respectively rate of predation by fish, respiratory rate of zooplankton, mortality rate of zooplankton,

Delay is frequently used in NPZ model to make the model biologically more realistic. Recently, researchers are using more than one delay to study the effect on the system populations. In the second phase of this study, i consider two delays in the model system (2.1). One discrete delay $\tau_{1}$ is considered in the specific growth rate of phytoplankton to incorporate the effect of gestation time which takes $\tau_{1}$ units of time to respond to changes in the phytoplankton population. The second delay $\tau_{2}$ is gestation time of zooplankton density. We thus obtain the following multi delayed $N P Z$ model

$$
\begin{aligned}
& \frac{d N}{d t}=I n-L_{r} N-\delta \frac{N P}{N+K_{p}}, \\
& \frac{d P}{d t}=\delta \frac{N\left(t-\tau_{1}\right) P}{N\left(t-\tau_{1}\right)+K_{p}}-r_{1} P-g_{s} \frac{P Z}{P+K_{Z}}, \\
& \frac{d Z}{d t}=g_{s} \frac{P\left(t-\tau_{2}\right) Z}{P\left(t-\tau_{2}\right)+K_{Z}}-r_{2} Z,
\end{aligned}
$$

where $\tau_{1}$ represents the gestation period of phytoplankton and $\tau_{2}$ gestation period of zooplankton respectively.

Feasibility The system (2.1) has only one interior equilibrium point given The equilibrium point $E^{*}$ which is biologically feasible if (i) $\left(g_{s}-r_{2}\right)>0$ and (ii) $\left(L_{r} k_{p}+\delta P^{*}-I_{n}\right)<0$. Linearizing the system (2.9) at $E^{*}$, we get

$$
\begin{aligned}
& \frac{d P}{d t}=a_{11} P(t)+a_{12} Z(t), \\
& \frac{d Z}{d t}=a_{22} Z(t)+a_{23} F(t)+b_{21} P\left(t-\tau_{1}\right), \\
& \frac{d F}{d t}=c_{32} Z\left(t-\tau_{2}\right),
\end{aligned}
$$

where $\quad a_{11}=-L_{r}-\delta \frac{P^{*}}{\left(N^{*}+K_{p}\right)^{2}}, \quad a_{12}=-\delta \frac{N^{*}}{N^{*}+K_{p}}, \quad a_{22}=$ $\delta \frac{P^{*} Z^{*}}{\left(P^{*}+K_{Z}\right)^{2}}, a_{23}=\delta \frac{P^{*}}{P^{*}+K_{Z}}, b_{21}=\delta \frac{K_{P} P^{*}}{\left(P^{*}+K_{P}\right)^{2}}, c_{32}=g_{s} \frac{Z^{*} K_{Z}}{\left(P^{*}+K_{Z}\right)^{2}}$. The corresponding characteristic equation is given by

$$
\lambda^{3}+A \lambda^{2}+B \lambda+C e^{-\lambda \tau_{1}} \lambda+e^{-\lambda \tau_{2}}(D \lambda+E)=0,
$$


where $\quad A=-\left(a_{11}+a_{22}\right), B=a_{11} a_{22}, C=-a_{12} b_{21}, D=$ $-a_{23} c_{32}$ and $E=a_{11} a_{23} c_{32}$. We now discuss the following cases.

Case I: $\tau_{1}=\tau_{2}=0$ For non-delayed system i.e., when $\tau_{1}=\tau_{2}=0$, characteristic equation (2.11) becomes

$\lambda^{3}+A \lambda^{2}+B \lambda+C \lambda+D \lambda+E=0$,

Hence, by Routh-Hurwitz criterion, we get the stability conditions of $E^{*}$

Theorem 1 The coexistence equilibrium point $E^{*}$ of system (2.1) is asymptotically stable if $A, B+C, D>0$ and $A(B+C)>D$.

Case II: $\tau_{1}>0, \tau_{2}=0$ We know that $i \omega(\omega>0)$ is a root of (2.11) if and only if $\omega$ satisfies

$-i \omega^{3}-A \omega^{2}+B i \omega+i C \omega\left(\cos \omega \tau_{1}-i \sin \omega \tau_{1}\right)$

$$
+D i \omega+E=0 \text {. }
$$

Separating real and imaginary parts, we get

$A \omega^{2}-E=C \omega \sin \omega \tau_{1}$,

$\omega^{3}-(B-D) \omega=C \omega \cos \omega \tau_{1}$.

These two equations give the positive values of $\tau$ and $\omega$ for which Eq. (2.11) can have purely imaginary roots. Squaring and adding these equations, we have

$\omega^{6}+p \omega^{4}+q \omega^{2}+r=0$,

where

$p=A^{2}-2(B+D)$,

$q=B+D-2 A E-C^{2}$,

$r=E^{2}$.

Now we shall measure the value of $\tau_{1}$ for which system (2.9) will remain stable. From Eq. (2.14) we have,

$\tau_{1_{k}}=\frac{1}{\omega_{k}}\left[\cos ^{-1}\left\{\frac{\omega_{k}^{3}-(B-D) \omega_{k}}{C \omega_{k}}\right\}+2 k \pi\right]$,

$k=0,1,2,3, \ldots$, where $\omega_{k}$ is the unique real positive root of Eq. (2.15). Also, differentiating Eq. (2.15) with respect to $\tau$ we get

$$
\left[\operatorname{Re}\left(\frac{d \lambda}{d \tau}\right)^{-1}\right]_{\omega=\omega_{0}, \tau=\tau_{0}}=\frac{U_{1} V_{1}+U_{2} V_{2}}{V_{1}^{2}+V_{2}^{2}}
$$

where

$$
\begin{aligned}
& U_{1}=-3 \omega_{0}^{2}+(B+D)-C \tau_{1_{0}} \cos \omega_{0} \tau_{1_{0}}, \\
& U_{2}=2 \omega_{0} A+C \tau_{1_{0}} \sin \omega_{0} \tau_{1_{0}}, \\
& V_{1}=\omega_{0} C \sin \omega_{0} \tau_{1_{0}}, \\
& V_{2}=\omega_{0} C \cos \omega_{0} \tau_{1_{0}} .
\end{aligned}
$$

Hence the transversality condition is satisfied if $\left(U_{1} V_{1}+U_{2} V_{2}\right)>0$.

Theorem 2 Assume $\tau_{1}>0, \tau_{2}=0$ and conditions of the Theorem 1 are satisfied, then the equilibrium $E^{*}\left(N^{*}, P^{*}, Z^{*}\right)$ is locally asymptotically stable for $\tau<\tau_{1_{0}}$ and unstable for $\tau>\tau_{1_{0}}$. Furthermore, the system (2.9) undergoes a Hopf bifurcation at $E^{*}\left(N^{*}, P^{*}, Z^{*}\right)$ when $\tau=\tau_{1_{0}}$, where

$\tau_{1_{0}}=\frac{1}{\omega_{0}}\left[\cos ^{-1}\left\{\frac{(A D-B C) \omega_{0}^{2}-C \omega_{0}^{4}}{D^{2}+C^{2} \omega_{0}^{2}}\right\}\right]$.

Case III: $\tau_{1}=\bar{\tau}_{1} \in\left(0, \tau_{1_{0}}\right), \tau_{2}>0$ Now we take $\tau_{1}=\bar{\tau}_{1} \in\left(0, \tau_{1_{0}}\right)$, then we know that $i \omega(\omega>0)$ is a root of (2.11) if and only if $\omega$ satisfies

$$
\begin{aligned}
& -i \omega^{3}-A \omega^{2}+B i \omega+i C \omega \cos \omega \bar{\tau}_{1}+C \omega \sin \omega \bar{\tau}_{1} \\
& \quad+D i \omega \cos \omega \tau_{2}+D \omega \sin \omega \tau_{2}-i E \sin \omega \tau_{2}+E \cos \omega \tau_{2}=0 .
\end{aligned}
$$

Separating real and imaginary parts, we get

$A \omega^{2}-C \omega \sin \omega \bar{\tau}_{1}=D \omega \sin \omega \tau_{2}+E \cos \omega \tau_{2}$,

$\omega^{3}-B \omega-C \omega \cos \omega \bar{\tau}_{1}=D \omega \cos \omega \tau_{2}-E \sin \omega \tau_{2}$.

These two equations give the positive values of $\tau_{2}$ and $\omega$ for which Eq. (2.11) can have purely imaginary roots. Squaring and adding these equations, we have

$\omega^{6}+p \omega^{4}+q \omega^{3}+r \omega^{2}+s=0$,

where

$p=A^{2}-2 B-2 C \cos \omega \bar{\tau}_{1}$,

$r=B^{2}+C^{2}-D^{2}+2 B C \cos \omega \bar{\tau}_{1}$,

$q=-2 A C \omega \sin \omega \bar{\tau}_{1}$,

$s=-E^{2}$.

Now we shall measure the value of $\tau_{2}$ for which system (2.9) will remain stable. From Eq. (2.31) we have,

$\tilde{\tau}_{2_{k}}=\frac{1}{\tilde{\omega}_{k}}\left[\cos ^{-1}\left\{\frac{\left(A E+B D-D C \cos \tilde{\omega}_{k} \bar{\tau}_{1}\right) \tilde{\omega}_{k}^{2}+D \tilde{\omega}_{k}^{4}-E C \tilde{\omega}_{k} \sin \tilde{\omega}_{k} \bar{\tau}_{1}}{E^{2}+D^{2} \tilde{\omega}_{k}^{2}}\right\}+2 k \pi\right]$, 
$k=0,1,2,3, \ldots$, where $\tilde{\omega}_{k}$ is the real positive root of Eq. (2.11). Also, differentiating Eq. (2.11) with respect to $\tau_{2}$ we get

$$
\left[\operatorname{Re}\left(\frac{d \lambda}{d \tau}\right)^{-1}\right]_{\omega=\tilde{\omega}_{0}, \tau=\tilde{\tau}_{2_{0}}}>0 .
$$

Hence the transversality condition is satisfied.

Theorem 3 Assume $\tau_{1}=\bar{\tau}_{1} \in\left(0, \tau_{1_{0}}\right), \tau>0$ and conditions of the Theorem 1 are satisfied, then the equilibrium $E^{*}\left(N^{*}, P^{*}, Z^{*}\right)$ is locally asymptotically stable for $\tau_{2}<\tilde{\tau}_{2_{0}}$ and unstable for $\tau_{2}>\tilde{\tau}_{2_{0}}$. Furthermore, the system (2.9) undergoes a Hopf bifurcation at $E^{*}\left(N^{*}, P^{*}, Z^{*}\right)$ when $\tau_{2}=\tilde{\tau}_{2_{0}}$, where

$$
\left[\operatorname{Re}\left(\frac{d \lambda}{d \tau}\right)^{-1}\right]_{\omega=\bar{\omega}_{0}, \tau=\tau_{2}}=\frac{U_{1} V_{1}+U_{2} V_{2}}{V_{1}^{2}+V_{2}^{2}}
$$

where

$$
\begin{aligned}
& U_{1}=-3 \bar{\omega}_{0}^{2}+B+C+\left(D-E \tau_{2_{0}}\right) \cos \bar{\omega}_{0} \tau_{2_{0}}-D \bar{\omega}_{0} \tau_{2_{0}} \sin \bar{\omega}_{0} \tau_{2_{0}}, \\
& U_{2}=-2 A \bar{\omega}_{0}+\left(D-E \tau_{2_{0}}\right) \sin \bar{\omega}_{0} \tau_{2_{0}}-D \bar{\omega}_{0} \tau_{2_{0}} \cos \bar{\omega}_{0} \tau_{2_{0}}, \\
& V_{1}=D \bar{\omega}_{0}^{2} \cos \bar{\omega}_{0} \tau_{2_{0}}-\bar{\omega}_{0} E \sin \bar{\omega}_{0} \tau_{2_{0}}, \\
& V_{2}=D \bar{\omega}_{0}^{2} \sin \bar{\omega}_{0} \tau_{2_{0}}+\bar{\omega}_{0} E \cos \bar{\omega}_{0} \tau_{2_{0}},
\end{aligned}
$$

Hence the transversality condition is satisfied if $\left(U_{1} V_{1}+U_{2} V_{2}\right)>0$.

$\tilde{\tau}_{2_{0}}=\frac{1}{\tilde{\omega}_{0}}\left[\cos ^{-1}\left\{\frac{\left(A E+B D-D C \cos \tilde{\omega}_{0} \bar{\tau}_{1}\right) \tilde{\omega}_{0}^{2}+D \tilde{\omega}_{0}^{4}-E C \tilde{\omega}_{0} \sin \tilde{\omega}_{0} \bar{\tau}_{1}}{E^{2}+D^{2} \tilde{\omega}_{0}^{2}}\right\}\right]$.

Case IV: $\tau_{1}=0, \tau_{2}>0$

We know that $i \omega(\omega>0)$ is a root of (2.11) if and only if $\omega$ satisfies

$$
\begin{aligned}
& -i \omega^{3}-A \omega^{2}+B i \omega+i C \omega+(D i \omega+E) \\
& \quad\left(\cos \omega \tau_{2}-i \sin \omega \tau_{2}\right)=0 .
\end{aligned}
$$

Separating real and imaginary parts, we get

$A \omega^{2}=E \cos \omega \tau_{2}+D \omega \sin \omega \tau_{2}$,

$\omega^{3}-(B+C) \omega=D \omega \cos \omega \tau_{2}-E \sin \omega \tau_{2}$.

These two equations give the positive values of $\tau_{2}$ and $\omega$ for which Eq. (2.11) can have purely imaginary roots. Squaring and adding these equations, we have

$\omega^{6}+p \omega^{4}+q \omega^{2}+r=0$,

where

$p=A^{2}-2(B+C)$,

$q=B^{2}+C^{2}-D^{2}$,

$r=-E^{2}$.

Now we shall measure the value of $\tau_{2}$ for which system (2.9) will remain stable. From Eq. (2.11) we have,

$\tau_{2_{k}}=\frac{1}{\tilde{\omega}_{k}}\left[\cos ^{-1}\left\{\frac{(E A-B D-C D) \tilde{\omega}_{k}^{2}+D \tilde{\omega}_{k}^{4}}{E^{2}+D^{2} \tilde{\omega}_{k}^{2}}\right\}+2 k \pi\right]$,

$k=0,1,2,3, \ldots$, where $\bar{\omega}_{k}$ is the real positive root of Eq. (2.24). Also, differentiating Eq. (2.11) with respect to $\tau_{2}$ we get
Theorem 4 Assume $\tau_{1}=0, \tau_{2}>0$ and conditions of the Theorem 1 are satisfied, then the equilibrium $E^{*}\left(N^{*}, P^{*}, Z^{*}\right)$ is locally asymptotically stable for $\tau<\tau_{2_{0}}$ and unstable for $\tau>\tau_{2_{0}}$. Furthermore, the system (2.9) undergoes a Hopf bifurcation at $E^{*}\left(N^{*}, P^{*}, Z^{*}\right)$ when $\tau=\tau_{2_{0}}$, where

$\tau_{2_{0}}=\frac{1}{\bar{\omega}_{0}}\left[\cos ^{-1}\left\{\frac{(A D-B C) \bar{\omega}_{0}^{2}-C \bar{\omega}_{0}^{4}}{D^{2}+C^{2} \bar{\omega}_{0}^{2}}\right\}\right]$.

Case $\quad V: \quad \tau_{2}=\hat{\tau}_{2} \in\left(0, \tau_{2_{0}}\right), \tau_{1}>0 \quad$ Now we take $\tau_{2}=\hat{\tau}_{2} \in\left(0, \tau_{2_{0}}\right)$, then we know that $i \omega(\omega>0)$ is a root of (2.11) if and only if $\omega$ satisfies

$$
\begin{aligned}
& -A \omega^{2}+C \omega \sin \omega \tau_{1}+D \omega \sin \omega \hat{\tau}_{2}+E \cos \omega \hat{\tau}_{2}-i \omega^{3} \\
& \quad+B i \omega+i C \omega \cos \omega \tau_{1}+i D \omega \cos \omega \hat{\tau}_{2}-i E \sin \omega \hat{\tau}_{2}=0 .
\end{aligned}
$$

Separating real and imaginary parts, we get

$-A \omega^{2}+D \omega \sin \omega \hat{\tau}_{2}+E \cos \omega \hat{\tau}_{2}=-C \omega \sin \omega \tau_{1}$,

$-\omega^{3}+B \omega+D \omega \cos \omega \hat{\tau}_{2}-E \sin \omega \hat{\tau}_{2}=-C \omega \cos \omega \tau_{1}$

These two equations give the positive values of $\tau_{2}$ and $\omega$ for which Eq. (2.11) can have purely imaginary roots. Squaring and adding these equations, we have

$\omega^{6}+p \omega^{4}+q \omega^{3}+r \omega^{2}+s=0$,

where 
$p=A^{2}-3 B-2 D \cos \omega \hat{\tau}_{2}$,

$r=B^{2}-C^{2}+D^{2}-2 A E \cos \omega \hat{\tau}_{2}+2 E \sin \omega \hat{\tau}_{2}$,

$q=-2 A D \sin \omega \hat{\tau}_{2}$,

$s=E^{2}$.

Now we shall measure the value of $\tau_{1}$ for which system (2.9) will remain stable. From Eq. (2.30) we have,

$$
\begin{aligned}
\bar{\tau}_{1_{k}}= & \frac{1}{\hat{\omega}_{k}}\left[\cos ^{-1}\left\{\frac{\hat{\omega}_{k}^{3}-B \hat{\omega}_{k}-D \hat{\omega}_{k} \cos \hat{\omega}_{k} \hat{\tau}_{2}+E \sin \hat{\omega}_{k} \hat{\tau}_{2}}{C \hat{\omega}_{k}}\right\}\right. \\
& +2 k \pi],
\end{aligned}
$$

$k=0,1,2,3, \ldots$, where $\hat{\omega}_{k}$ is the real positive root of Eq. (1.31). Also, differentiating Eq. (2.11) with respect to $\tau_{1}$ we get

$$
\left[\operatorname{Re}\left(\frac{d \lambda}{d \tau}\right)^{-1}\right]_{\omega=\hat{\omega}_{0}, \tau_{1}=\bar{\tau}_{1_{0}}}=\frac{u_{1} v_{1}+u_{2} v_{2}}{v_{1}^{2}+v_{2}^{2}},
$$

where

$$
\begin{aligned}
u_{1}= & C+\sin \hat{\omega}_{0} \tau \bar{\tau}_{1_{0}}\left(-2 A \hat{\omega}_{0}+\bar{\tau}_{1_{0}} \hat{\omega}_{0}^{3}-B \bar{\tau}_{1_{0}} \hat{\omega}_{0}\right) \\
& +\cos \hat{\omega}_{0} \bar{\tau}_{1_{0}}\left(B-3 \hat{\omega}_{0}^{2}-A \bar{\tau}_{1_{0}} \hat{\omega}_{0}^{2}\right) \\
u_{2}= & \sin \hat{\omega}_{0} \bar{\tau}_{1_{0}}\left(B-3 \hat{\omega}_{0}^{2}-A \bar{\tau}_{1_{0}} \hat{\omega}_{0}^{2}\right) \\
& +\cos \hat{\omega}_{0} \bar{\tau}_{1_{0}}\left(2 A \hat{\omega}_{0}-\bar{\tau}_{1_{0}} \hat{\omega}_{0}^{3}+B \bar{\tau}_{1_{0}} \hat{\omega}_{0}\right) \\
v_{1}= & -A \hat{\omega}_{0}^{3} \sin \hat{\omega}_{0} \bar{\tau}_{1_{0}}+\cos \hat{\omega}_{0} \bar{\tau}_{1_{0}}\left(B \hat{\omega}_{0}^{2}-\hat{\omega}_{0}^{4}\right) \\
v_{2}= & \sin \hat{\omega}_{0} \bar{\tau}_{1_{0}}\left(B \omega^{2}-\omega^{4}\right)+A \omega^{3} \cos \omega \bar{\tau}_{1_{0}} .
\end{aligned}
$$

Hence the transversality condition is satisfied if $\left(U_{1} V_{1}+U_{2} V_{2}\right)>0$.

Theorem 5 Assume $\tau_{2}=\hat{\tau}_{2} \in\left(0, \tau_{2_{0}}\right), \tau_{1}>0$ and conditions of the Theorem 1 are satisfied, then the equilibrium $E^{*}\left(N^{*}, P^{*}, Z^{*}\right)$ is locally asymptotically stable for $\tau_{1}<\bar{\tau}_{1_{0}}$ and unstable for $\tau_{1}>\bar{\tau}_{1_{0}}$. Furthermore, the system (2.9) undergoes a Hopf bifurcation at $E^{*}\left(N^{*}, P^{*}, Z^{*}\right)$ when $\tau_{1}=\bar{\tau}_{1_{0}}$, where

$$
\bar{\tau}_{1_{0}}=\frac{1}{\hat{\omega}_{0}}\left[\cos ^{-1}\left\{\frac{(A D-B C) \hat{\omega}_{0}^{2}-C \hat{\omega}_{0}^{4}}{D^{2}+C^{2} \hat{\omega}_{0}^{2}}\right\}\right] .
$$

\section{Numerical simulation}

In Fig. 1, we present the geographical position of the study area which is briefly describe in "Study area" section. Now we consider the parameter set of the system as: $I_{n}=1.140$, $L_{r}=0.45, \quad K_{p}=12, \quad r_{p h}=0.1090, \quad r_{s}=0.049$, $M_{r p}=0.021, g=0.2041, \quad s_{e}=7.4, \quad s_{r}=7.2, \quad K_{z}=65$, $r_{z o}=0.008, \quad r_{f p}=0.001, \quad M_{r z}=0.0125, \quad V=1.60$, $p_{\max }=2.62, T=28.1, E_{c}=0.2, d=4, I_{o p t}=680$ and $I_{r}=800$. Then the equilibrium population density is $E^{*}(2.96278,1.7912,5.62218)$. In Fig. 2 we show the (a) $\mathrm{T}=25.39^{\circ} \mathrm{C}, \mathrm{s}_{\mathrm{e}}=12.17 \mathrm{ppt}, \mathrm{s}_{\mathrm{r}}=6.37 \mathrm{ppt}$

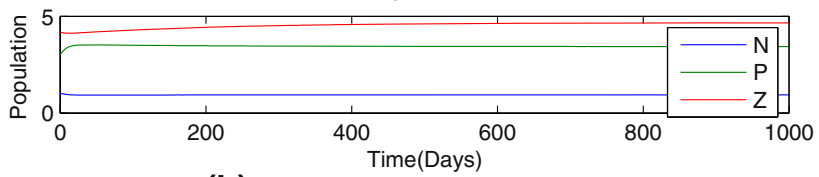

(b) $\mathrm{T}=23.38^{\circ} \mathrm{C}, \mathrm{s}_{\mathrm{e}}=12.17 \mathrm{ppt}, \mathrm{s}_{\mathrm{r}}=6.37 \mathrm{ppt}$

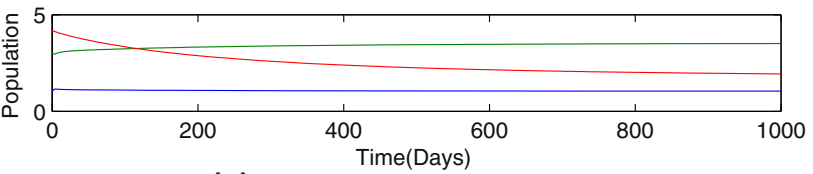

(C) $\mathrm{T}=12.1^{\circ} \mathrm{C}, \mathrm{s}_{\mathrm{e}}=12.17 \mathrm{ppt}, \mathrm{s}_{\mathrm{r}}=6.37 \mathrm{ppt}$

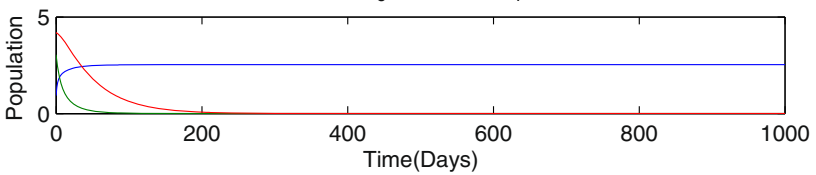

Fig. 2 Time series of the NPZ dynamics for different parameter conditions in Hooghly-Matla estuarine system. The model results are obtained by varying the values of temperature with fixed salinity effect. a Temperature $(T)=25.39^{\circ} \mathrm{C}$, salinity of downstream $\left(S_{e}\right)=12.17$, salinity of upstream $\left(S_{r}\right)=6.37 ; \mathbf{b} T=23.38^{\circ} \mathrm{C}, S_{e}=$ $12.17, S_{r}=6.37 ; \mathbf{c} T=12.1^{\circ} \mathrm{C}, S_{e}=12.17, S_{r}=6.37$

(a) $\mathrm{T}=25.39^{\circ} \mathrm{C}, \mathrm{s}_{\mathrm{e}}=12.17 \mathrm{ppt}, \mathrm{s}_{\mathrm{r}}=6.37 \mathrm{ppt}$

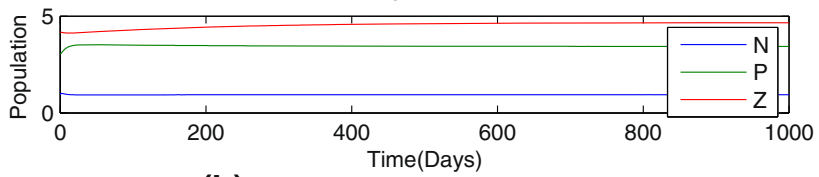

(b) $\mathrm{T}=25.39^{\circ} \mathrm{C}, \mathrm{s}_{\mathrm{e}}=12.17 \mathrm{ppt}, \mathrm{s}_{\mathrm{r}}=4.00 \mathrm{ppt}$

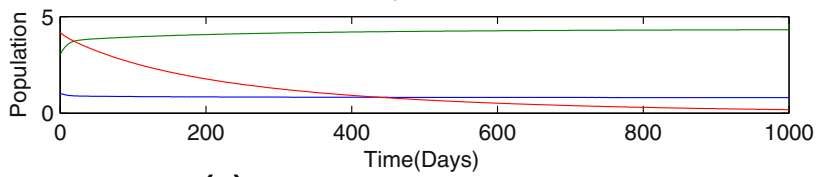

(C) $\mathrm{T}=25.39^{\circ} \mathrm{C}, \mathrm{s}_{\mathrm{e}}=12.17 \mathrm{ppt}, \mathrm{s}_{\mathrm{r}}=2.25 \mathrm{ppt}$

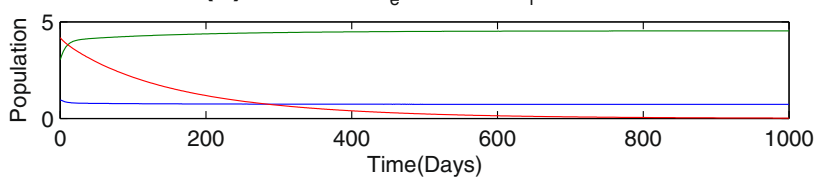

Fig. 3 Time series of the NPZ dynamics during different conditions in Hooghly-Matla estuarine system. The model results are obtained by varying the values of salinity parameter with fixed temperature. a $T=25.38^{\circ} \mathrm{C}, S_{e}=12.17, S_{r}=6.37 ; \mathbf{b}$ Temperature $(T)=25.38^{\circ} \mathrm{C}$, salinity of downstream $S_{e}=12.17$, salinity of upstream $S_{r}=4$; c $T=25.38^{\circ} \mathrm{C}, S_{e}=12.17, S_{r}=2.25$

temperature effect on the dynamics of non-delayed system 2.1 by plotting the time series. Here we observe that when temperature is almost $23.38-25.39{ }^{\circ} \mathrm{C}$, coexistence equilibrium point $E^{*}$ is stable (Fig. 2a, b), i.e., nutrient $(N)$, phytoplankton $(P)$ and zooplankton $(Z)$ coexist together. But if temperature decreases, then rate of phytoplankton bloom decreases and eventually due to lack of food zooplankton abundance decreases. At $12.1^{\circ} \mathrm{C}$, we see both 
Fig. 4 Time series (i) and phase space (ii) diagram of system (2.1), figures show that system (2.1) is asymptotically stable. Other parameters are as in the text

Fig. 5 Time series (i, iiI) and phase space (ii, iv) diagram of system (2.9) when

$\tau_{1}>0, \tau_{2}=0$, $\mathbf{i}$ and ii show that system (2.9) is stable for $\tau_{1}=0.5<\tau_{1_{0}}=2.44, \tau_{2}=0$ and iii and iv show that system (2.9) is unstable for $\tau_{1}=$ $2.7>\tau_{1_{0}}=2.44, \tau_{2}=0$ respectively. Other parameters are as in the text

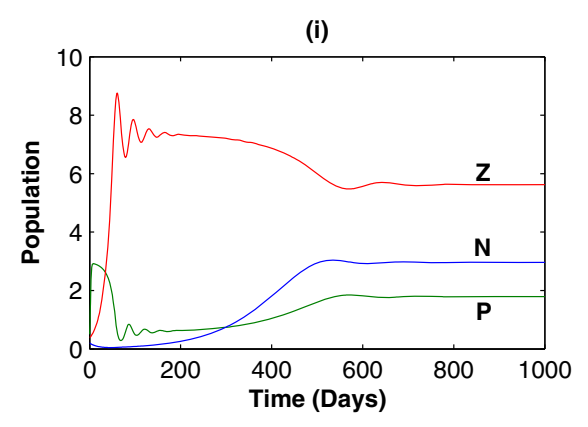

(ii)

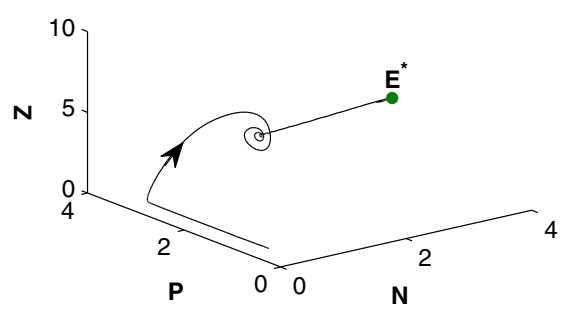

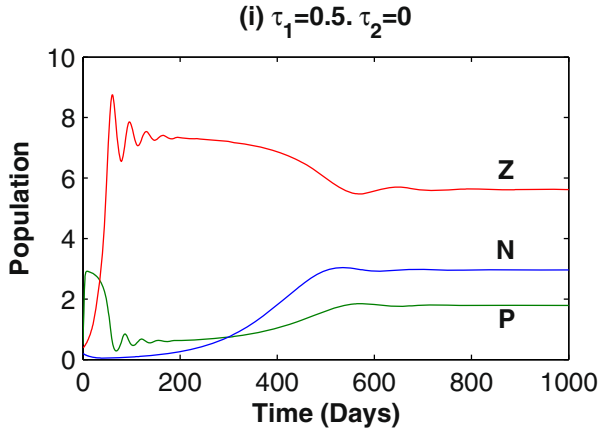

(ii)

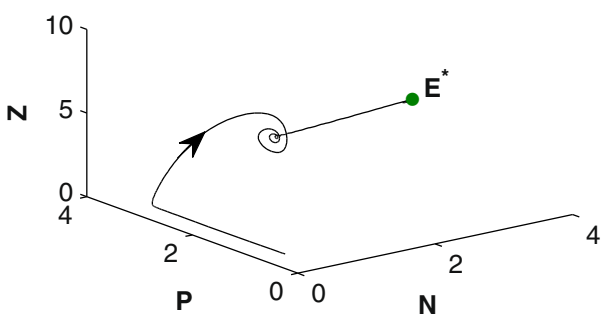

(iii) $\tau_{1}=2.7, \tau_{2}=0$

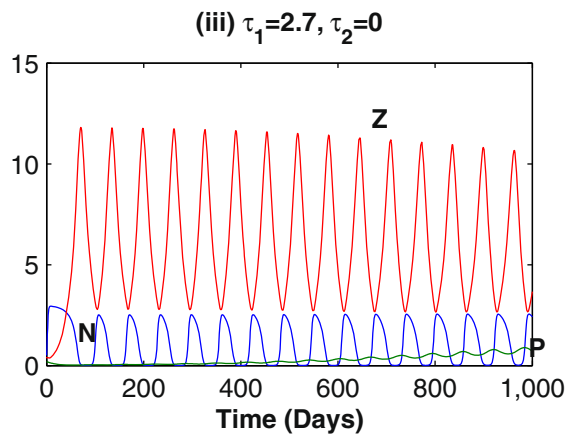

(iv)

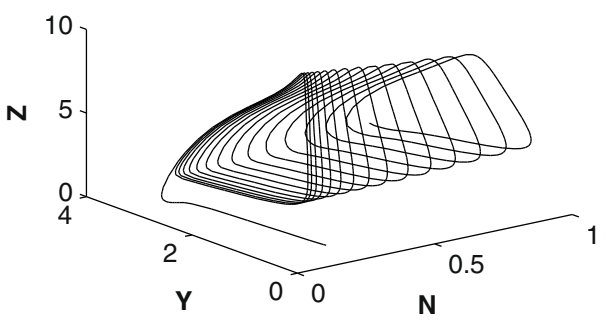

phytoplankton and zooplankton go to zero (Fig. 2c). So, from these three figures, it is very clear that temperature dependent phytoplankton growth rate plays a very significant role to maintain the stability of coexistence equilibrium point $E^{*}$. Figure 3 (time series) shows that at higher salinity three species coexist (Fig. 3a, b) and at lowest salinity zooplankton goes to zero (Fig. 3c). So it is clear from the figure that decreasing salinity decreases the grazing rate of zooplankton and lack of low grazing rate zooplankton gradually goes to zero. Figure 4 [Time series (i) and phase space (ii)] shows that system (2.1) i.e. the non-delayed ( $\tau_{1}=\tau_{2}=0$ ) (Case I) system of system (2.9) is stable. Now if we take $\tau_{1}>0, \tau_{2}=0$ (Case II), then Fig. 5(i) (time series) and (ii) (phase space) show that system (2.9) is stable for $\tau_{1}=0.5<\tau_{1_{0}}=2.44, \tau_{2}=0$ and Fig. 5(iii) (time series) and (iv) (phase space) show that system (2.9) is unstable for $\tau_{1}=0.5<\tau_{1_{0}}=2.44, \tau_{2}=0$ respectively. If we take $\tau_{1}=0.5=\bar{\tau}_{1} \in\left(0, \tau_{1_{0}}\right), \tau_{2}>0$ (Case III), then Fig. 6(i) (time series) and (ii) (phase space) show that system (2.9) is stable for $\tau_{1}=0.5, \tau_{2}=$ $2.1>\tilde{\tau}_{2_{0}}=1.7$ and Fig. 6(iii) (time series) and (iv) (phase space) show that system (2.9) is unstable for $\tau_{1}=0.5, \tau_{2}=$ $1.57<\tilde{\tau}_{2_{0}}=1.7$ respectively. Again if we take $\tau_{1}=$ $0, \tau_{2}>0$ (Case IV), then Fig. 7(i) (time series) and (ii) (phase space) show that system (2.9) is stable for $\tau_{1}=$ $0, \tau_{2}=0.3<\tau_{2_{0}}=1.57$ and Fig. 7(iii) (time series) and (iv) (phase space) show that system (2.9) is unstable for $\tau_{1}=0, \tau_{2}=1.9>\tau_{2_{0}}=1.57$ respectively. Also when we take $\tau_{1}>0, \tau_{2}=0.3=\hat{\tau}_{2} \in\left(0, \tau_{2_{0}}\right) \quad($ Case $\mathrm{V})$, then Fig. 8(i) (time series) and (ii) (phase space) show that system (2.9) is stable for $\tau_{1}=1.5<\bar{\tau}_{1_{0}}, \tau_{2}=0.3$ and Fig. 8(iii) (time series) and (iv) (phase space) show that system (2.9) is unstable for $\tau_{1}=2.5>\bar{\tau}_{1_{0}}, \tau_{2}=0.3$ respectively. 


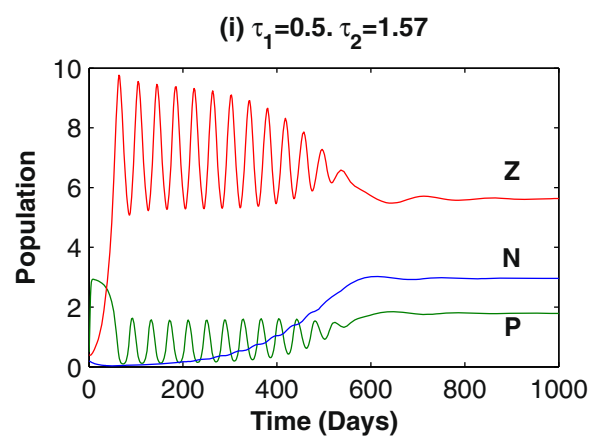

(ii)

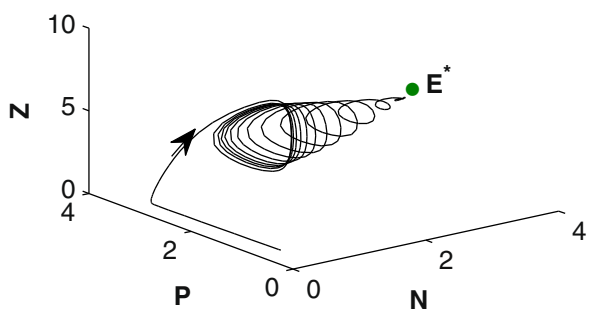

Fig. 6 Time series (i, ii) and phase space (ii, iv) diagram of system (2.9) when $\tau_{1}=0.5=\bar{\tau}_{1} \in\left(0, \tau_{1_{0}}\right), \tau_{2}>0$, $\mathbf{i}$ and $\mathbf{i i}$ show that system (2.9) is stable for $\tau_{1}=0.5, \tau_{2}=1.57<\tilde{\tau}_{2_{0}}=1.7$ and iii and iv show

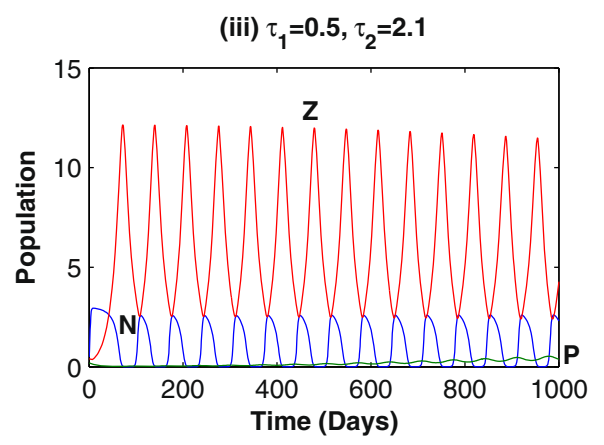

(iv)

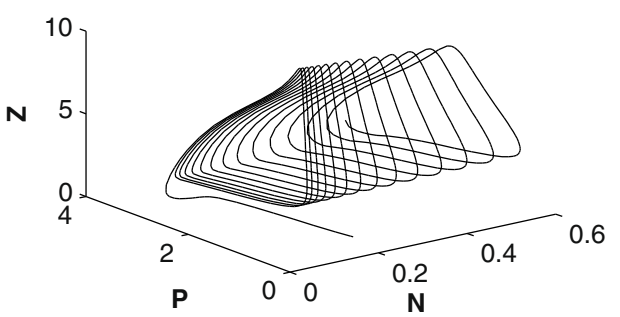

that system (2.9) is unstable for $\tau_{1}=0.5, \tau_{2}=2.1>\tilde{\tau}_{2_{0}}=1.7$ respectively. Other parameters are as in the text

Fig. 7 Time series (i, ii) and phase space (iii, iv) diagram of system (2.9) when

$\tau_{1}=0, \tau_{2}>0, \mathbf{i}$ and $\mathbf{i i}$ show that system (2.9) is stable for $\tau_{1}=0, \tau_{2}=0.3<\tau_{2_{0}}=1.57$ and iii and iv show that system (2.9) is unstable for $\tau_{1}=0, \tau_{2}=$ $1.9>\tau_{2_{0}}=1.57$ respectively. Other parameters are as in the text

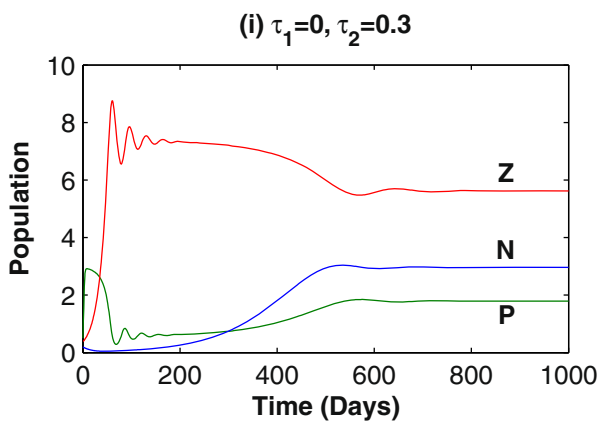

(ii)

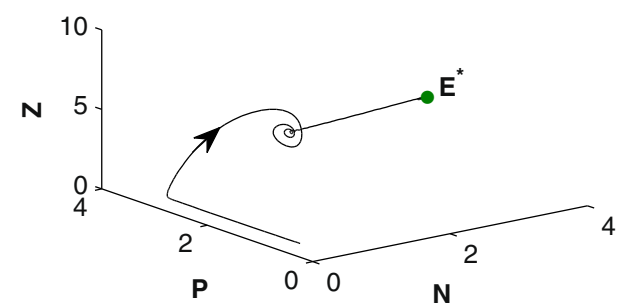

(iii) $\tau_{1}=0, \tau_{2}=1.9$

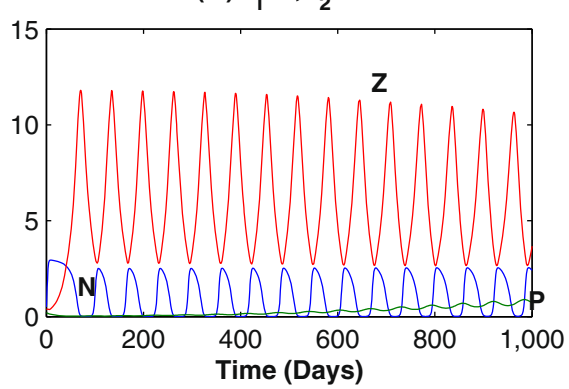

(iv)

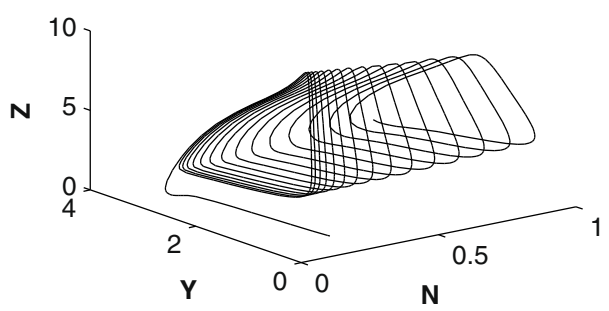

\section{Discussion}

In this present work, we consider a NPZ-model with Holling type II functional response where photosynthesis rate of phytoplankton, salinity induced grazing rate of zooplankton and gestation delay of both phytoplankton and zooplankton population independently are considered. In the later part of the study, we show the analytical results of the impact of delay on the stability of the system and quantitative results of the Hopf bifurcation. Delay induced deterministic system shows that there is a critical value of delay parameter bellow which system is stable and above which system is unstable and when the delay parameter crosses the critical value then system experiences Hopfbifurcation. Also all the results are compared and validated by numerical examples with real data set. 


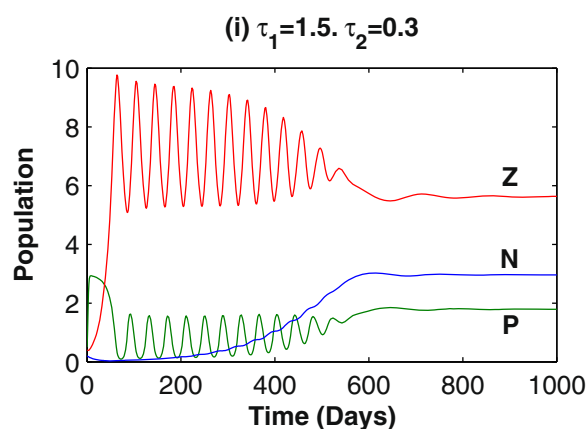

(ii)

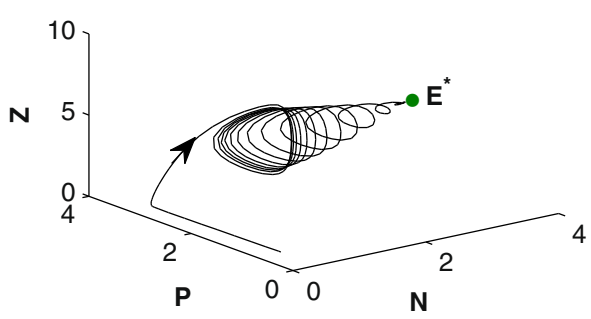

Fig. 8 Time series (i, iii) and phase space (ii, iv) diagram of system (2.9) when $\tau_{1}>0, \tau_{2}=0.3=\hat{\tau}_{2} \in\left(0, \tau_{2_{0}}\right)$, $\mathbf{i}$ and $\mathbf{i i}$ show that system (2.9) is stable for $\tau_{1}=1.5<\bar{\tau}_{1_{0}}, \tau_{2}=0.3$ and iii and iv show that

The main purpose of this work is to observe the effect of multiple time delays along with photosynthesis rate of phytoplankton, salinity induced grazing rate of zooplankton on a NPZ-system. Results show that these biological parameters play a significant role to measure the critical value of delay parameter/parameters which is/are the key factor to maintain the stability of the system. It is quite natural that when delay parameter/parameters is/are small enough the system can persist its stability but for large delay scale, system becomes unstable. But delay parameter/parameters can not effect the equilibrium population abundance.

The model is run for the time period of 1000 days and the dynamics of $N P Z$ is predicted by the model. The phytoplankton and zooplankton population will go extinct rather nutrient value remain uniform after certain passage of time (days) when the temperature is $<12.1{ }^{\circ} \mathrm{C}$ and there is a constant difference between $S_{e}$ and $S_{r}$ in the estuarine system (Fig. 2a). Figure 2a shows that the nutrient is abundant in the system for a long time but the phytoplankton and zooplankton population eliminate after 100 and 200 days respectively. The physical conditions of the environment are not favorable for the growth of phytoplankton as a result phytoplankton population do exist in the system for a very short period of time and then eliminated from the system. Figure $2 b$ shows a little improvement in the phytoplankton population but there is no improvement of zooplankton population in the system. Since the zooplankton population is dependent on the

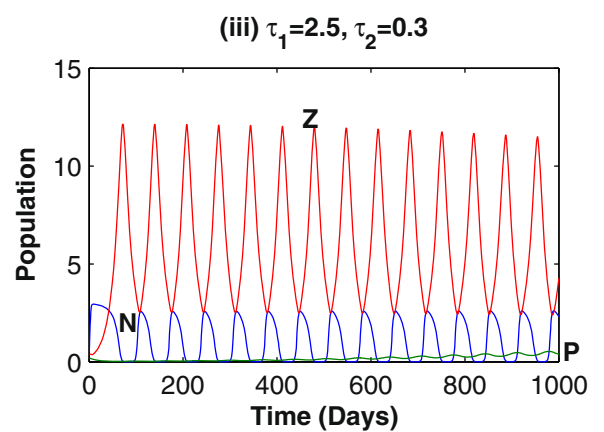

(iv)

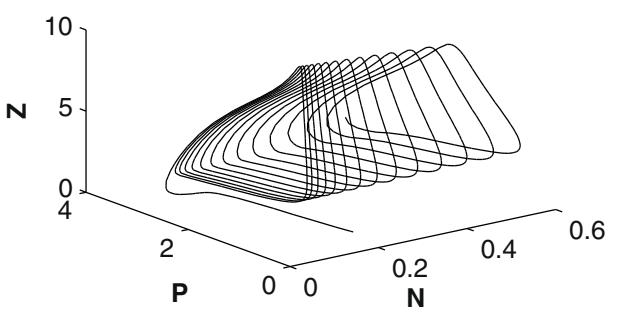

system (2.9) is unstable for $\tau_{1}=2.5>\bar{\tau}_{1_{0}}, \tau_{2}=0.3$ respectively. Other parameters are as in the text

phytoplankton population, the phytoplankton population grows first with gradual increase of temperature and little after the phytoplankton population. When the temperature $\mathrm{T}$ is fixed at $23.38^{\circ} \mathrm{C}$, it is shows the optimum growth for both plankton in this coastal environment. Our model shows that there is a definite role of temperature in the growth rate of zooplankton (Fig. 2c). When the temperature is raised to 23.38 and $25.38^{\circ} \mathrm{C}$. the system shows a resilient condition, where the zooplankton population put itself back to its existence in this environmental condition along with phytoplankton and available nutrient(cf. Fig. 2c). The model also describes a situation where, there is a little difference in $\mathrm{S}_{\mathrm{e}}$ and $S_{r}$ (monsoon season-July to October in the tropical region) of the estuary. At $25.39^{\circ} \mathrm{C}$, temperature all the components (NPZ) coexist and a stable equilibrium is attained by the system. These oscillations are continued to exist for a long time if the value of $S_{e}$ approaches to $S_{r}$, when fresh water run off is more in the estuary for a long time. The above observation show that the salinity is an important factor governing the plankton dynamics in the estuary.

Model also shows that the salinity induced grazing of zooplankton $\left(g_{s}\right)$ should be greater the loss due to respiration, mortality and predation $\left(r_{2}\right)$, for the existence of the system. During the pre-monsoon period, the grazing pressure of zooplankton on phytoplankton is maximum; and the environmental conditions are favorable for primary production. In Hooghy-Matla estuarine system, fish population rise during monsoon. During pre-monsoon, the predation 
pressure of carnivore fishes on zooplankton and herbivore fishes on phytoplankton is less in comparison to monsoon period; this situation favours the coexistence of phytoplankton and zooplankton population in the system (Mandal et al. 2012).

From the study of the system in that way, temperature dependent phytoplankton growth rate and salinity induced grazing rate of zooplankton are the key factors to describe the dynamical behavior of the system. For this reason, all the critical values of delay parameters are functions of temperature dependent phytoplankton growth rate and salinity induced grazing rate of zooplankton. Temperature and salinity effect on the system is very clearly described by Ray and Straskraba (2001), Mandal et al. (2012). In Mandal et al. (2012), authors show the temperature and salinity effect on phytoplankton growth rate and zooplankton grazing rate by real date set on the Hoogly-Matla estuary system as study area and they can plot the graph time functions of these two important system parameters. Basis on these results, we try to show the effect of gestation delays of phytoplankton and zooplankton on this system. Naturally, delay parameters become temperature and salinity dependent. So stable and unstable dynamics through Hopf bifurcation are strictly temperature and salinity dependent. It is very difficult to make proper time dependent function of temperature dependent phytoplankton growth rate and salinity induced zooplankton grazing rate from the graph time function of Mandal et al. (2012). For this reason non-autonomous system analysis is not possible, so better choice to describe such a system in numerical way. Most important motivation of this study is to describe the critical values of the delay parameters as a function of temperature and salinity intensity. So, the study is very relevant to describe that type of system and also to describe the significance of delay through the literature of ecology and mathematics.

\section{References}

Baird ME, Emsley SM, McGlade JM (2001) Modelling the interacting effects of nutrient uptake, light capture and temperature on phytoplankton growth. J Plankton Res 23:829-840

Berryman AA (1992) The origins and evolution of predatorprey theory. Ecology 73:1530-1535

Bintz JC, Nixon SW, Buckley BA, Granger SL (2003) Impacts of temperature and nutrients on coastal lagoon plant communities. Estuaries 26:765-776

Bissinger JE, Montagnes DJS, Sharples JS, Atkinson D (2008) Predicting marine phytoplankton maximum growth rates from temperature: improving on the Eppley curve using quantile regression. Limnol Oceanogr 53:487-493

Calbet A, Saiz E (2005) The ciliate-copepod link in marine ecosystems. Aquat Microb Ecol 38:157-167
Cottingham KL, Glaholt S, Brown AC (2004) Zooplankton community structure affects how phytoplankton respond to nutrient pulses. J Ecol 85:158-171

Di Toro DM, O'Connor DJ, Thomann RV, Mancini JL (1975) Phytoplankton-zooplankton-nutrient interaction model for Western Lake Erie. In: Pattern BC (ed) Systems analysis and simulation in ecology, vol 3. Academic Press Inc., NY, pp 423-474

Dube A, Jayaraman G (2008) Mathematical modelling of the seasonal variability of plankton in a shallow lagoon. J Nonl Anal 69:850-865

Dube A, Jayaraman G, Rani R (2010) Modelling the effects of variable salinity on the temporal distribution of plankton in shallow coastal lagoons. J Hydro-environment Res 4:199-209

Flynn KJ (2003) Modelling multi-nutrient interactions in phytoplankton; balancing simplicity and realism. Prog Oceanogr 56:249-279

Ghosh PB (2001) Role of macrofauna in energy partitioning and nutrient recycling in a tidal creek of Sunderbans mangrove forest, India. In: Kumar A (ed) Ecology and ethology of aquatic biota. Daya Publishing House, New Delhi, pp 90-97

Hassell MP (1971) Mutual interference between searching insect parasites. J Anim Ecol 40:473-86

Irigoien X, Flynn KJ, Harris RP (2005) Phytoplankton blooms: a 'loophole' in microzooplankton grazing impact. J Plankton Res 27:313-321

Jana D (2014) Stabilizing Effect of prey refuge and predator's interference on the dynamics of prey with delayed growth and generalist predator with delayed gestation. Int J Ecol 2014:12, Article ID 429086. doi:10.1155/2014/429086

Jana D, Agrawal R, Upadhyay RK (2014) Top-predator interference and gestation delay as determinants of the dynamics of a realistic model food chain. Chaos Solitons Fractals 69:50-63

Jana D, Agrawal R, Upadhyay RK (2015) Dynamics of generalist predator in a stochastic environment: effect of delayed growth and prey refuge. Appl Math Comput 268:1072-1094

Jana D, Pathak R, Agarwal M (2016) On the stability and Hopf bifurcation of a prey-generalist predator system with independent age-selective harvesting. Chaos Solitons Fractals $83: 252-273$

Jana D, Samanta GP (2014) Role of multiple delays in ratiodependent prey-predator system with prey harvesting under stochastic environment. Neural Parallel Sci Comput 22:205-222

Ketchum BH (1951) The exchange of fresh and salt water in tidal estuaries. J Mar Res 10:1838

Lomas MW, Trice TM, Glibert PM, Bronk DA, Mc Carthy JJ (2002) Temporal and spatial dynamics of urea uptake and regeneration rates and concentrations in Chesapeake Bay. Estuaries 25:469-482

MacDonald M (1989) Biological delay systems: linear stability theory. Cambridge University Press, Cambridge

Mandal S, Ray S, Ghose PB (2012) Modelling nutrint (dissolved inorganic nitrogen) and plankton dynamics at sagar island of Hooghly-Matla estuarine system, West Bengal, India. J Nat Resour Model 25(4):629-653

May RM (1981) Theoretical ecology: principles and applications. Blackwell Scientific Publications, Oxford

Morozov AY, Nikolay PN, Sergei VP (2005) Invasion of a top predator into an epipelagic system can bring a paradoxical topdown trophic control. Biol Invasions 7:845-861

Nicklisch A, Shatwell T, Kohler J (2008) Analysis and the modelling of the interactive effects of temperature and light on phytoplankton growth and relevance for the spring bloom. J Plankton Res 30:75-91

Ray S, Straskraba M (2001) The impact of detritivorous fishes on a mangrove estuarine system. Ecol Model 140:207-218 
Ray S, Berec L, Straskraba M, Jorgensen SE (2001) Optimization of exergy and implications of body sizes of phytoplankton and zooplankton in an aquatic ecosystem model. J Ecol Model 140:219-234

Radtke E, Straskraba M (1980) Self-optimization in a phytoplankton model. Ecol Model 9:247-268

Ruan S (2009) On nonlinear dynamics of predator-prey models with disc rete delay. Math Model Nat Phenom 4(2):140-188

Sih A et al (1985) Predation, competition, and prey communities, a review of field experiments. Annu Rev Ecol Syst 16:269-311
Sipkay C (2010) Modelling of the possible effect of climate change based on a Danubian phytoplankton database. Doctoral dissertation, Corvinus University of Budapest

Smayda TJ (2004) Eutrophication and phytoplankton. In: Wassmann $\mathrm{P}$, Olli K (eds) Integrated approaches to drainage basin nutrient inputs and coastal eutrophication, pp 89-98. Electronic-Book (pdf file on web page: http://www.ut.ee/olli/eutr/)

Zhou YA (2006) Nutrient-phytoplankton-zooplankton model for classifying estuaries based on susceptibility to nitrogen loads, M.Sc. Dissertation, University of Michigan 